\title{
Radiative Properties Estimation and Construction of Confidence Regions with a Combination of the Differential Evolution Algorithm and the Likelihood Method
}

\author{
Diego C. Knupp ${ }^{1}$ \\ João Vítor M. Canato \\ Antônio J. Silva Neto ${ }^{3}$ \\ Departamento de Engenharia Mecânica e Energia, IPRJ/UERJ, Nova Friburgo, RJ \\ Francisco J. C. P. Soeiro ${ }^{4}$ \\ Departamento de Engenharia Mecânica, FEN/UERJ, Rio de Janeiro, RJ
}

\begin{abstract}
This work is aimed at the combination of the Differential Evolution algorithm and the likelihood method for the estimation of radiative properties and construction of confidence regions of the parameters estimates. Two cases with different levels of measurement error are employed, and the results indicate that the approach is adequate for the construction of confidence regions in the radiative transfer inverse problem considered. The results also demonstrate that with increasing measurement errors the traditionally employed elliptical confidence region might lead to poor approximations in this problem.
\end{abstract}

Keywords. Inverse problems, Radiative transfer, Confidence regions, Likelihood method

\section{Introduction}

The analysis of direct and inverse radiative transfer problems in participating media has several practical applications, including optical tomography, computerized tomography, hydrologic optics and radiative properties estimation $[1,2]$. The inverse problem is classically formulated through the Maximum Likelihood approach, leading to an objective function to be minimized. For the optimization problem, even though gradient based methods have been successfully employed in several contexts, such as the LevenbergMarquardt method, stochastic methods have gained popularity with the improvement in computational speed of modern computers, as reviewed in [2].

These stochastic algorithms, in general, mimic some natural optimization behaviors found in nature and are designed to find an approximation of the global optimum of a given objective function through extensive calculation at several points. These algorithms are specially attractive for high dimensional problems and objective functions with several

\footnotetext{
${ }^{1}$ diegoknupp@iprj.uerj.br

2jvmcanato@gmail.com

3 ajsneto@iprj.uerj.br

${ }^{4}$ soeiro@uerj.br
} 
local minima, as they are not very sensitive to the initial parameters guesses, contrary to gradient based methods that might converge to a local minimum close to the initial guess [2]. The main drawback generally seen in these methods is the high number of function evaluations needed, but Schwaab and co-workers [3] have shown that these solution points generated during the iterative procedure can actually be employed for the construction of more reliable confidence regions of the parameters estimates in comparison with the traditionally employed elliptical confidence region, which might constitute a very poor approximation in nonlinear inverse problems [4].

This work is aimed at the inverse radiative transfer problem solution with the stochastic method known as the Differential Evolution algorithm [5] for the estimation of the optical thickness, single scattering albedo and diffuse reflectivity coefficients in one-dimensional participating media, and construction of the confidence regions of the sought parameters through the likelihood method. The results are critically compared against the frequently used elliptical confidence region for different levels of measurement error.

\section{Problem Formulation and Solution Methodology}

Consider a one-dimensional participating medium with boundaries at $\tau=0$ and $\tau=\tau_{0}$ that reflect diffusely the radiation that comes from inside the medium. The boundary surfaces are subjected to the incidence of radiation originated at external sources with intensities $F_{1}$ and $F_{2}$ at $\tau=0$ and $\tau=\tau_{0}$, as shown in Figure 1 .

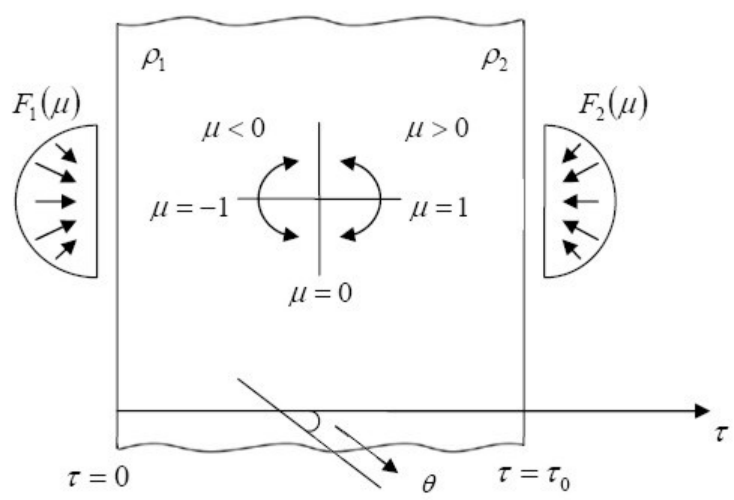

Figure 1: Schematic representation of the one-dimensional participating medium.

The mathematical model for the interaction of the radiation with the participating medium considering no emission, isotropic scattering and azimuthal symmetry is given by the linear version of the Boltzmann equation [1], given below in its dimensionless form:

$$
\mu \frac{\partial I(\tau, \mu)}{\partial \tau}+I(\tau, \mu)=\frac{\omega}{2} \int_{-1}^{1} I\left(\tau, \mu^{\prime}\right) d \mu^{\prime}, \quad 0<\tau<\tau_{0}, \quad-1 \leq \mu \leq 1
$$




$$
\begin{aligned}
& I(0, \mu)=F_{1}+2 \rho_{1} \int_{0}^{1} I\left(0,-\mu^{\prime}\right) \mu^{\prime} d \mu^{\prime}, \quad \mu>0 \\
& I\left(\tau_{0}, \mu\right)=F_{2}+2 \rho_{2} \int_{0}^{1} I\left(\tau_{0}, \mu^{\prime}\right) \mu^{\prime} d \mu^{\prime}, \quad \mu<0
\end{aligned}
$$

where $I$ represents the radiation intensity, $\tau$ is the optical variable, $\mu$ is the cosine of the polar angle $\theta$ (angle formed between the radiation beam and the $\tau$ axis), $\omega$ is the single scattering albedo, and $\rho_{1}$ and $\rho_{2}$ are the diffuse reflectivities at $\tau=0$ and $\tau=\tau_{0}$, respectively. For the solution of Eqs. (1a-1c) the polar angle domain is discretized and the integral term on the RHS of Eq. (1a) is substituted by a gaussian quadrature, leading to a system of ODE's which are numerically solved through the NDSolve routine of the Mathematica software, under automatic absolute and relative error control, with iterative forward and backward sweeps (from $\tau=0$ to $\tau=\tau_{0}$, and from $\tau=\tau_{0}$ to $\tau=0$ ) until convergence is achieved. The Mathematica routine then provides an interpolation function object that approximates the $\tau$ and $\mu$ behaviors of the solution in a continuous form in such a way that the calculated intensity $I$ can be obtained in any desired position and polar angle.

\section{Inverse Analysis}

Suppose the following vector of radiative properties is unknown:

$$
\mathbf{Z}=\left\{\tau_{0}, \omega, \rho_{1}, \rho_{2}\right\}
$$

Nonetheless, experimental data on the radiation that leaves the medium may be available. For instance, these measurements can be acquired in different polar angles with external detectors located at $\tau=0$ and $\tau=\tau_{0}$, here denoted by $Y_{i}, i=1,2, \ldots, N_{d}$, where $N_{d}$ is the number of experimental measurements available.

Considering the measurement errors related to the data $\mathbf{Y}$ are additive and have normal distribution with zero mean and covariance matrix given by $\mathbf{W}$, the probability density for the occurrence of the measurements $\mathbf{Y}$ with the given parameters values $\mathbf{Z}$ can be expressed as [6]:

$$
\pi(\mathbf{Y} \mid \mathbf{Z})=(2 \pi)^{-N_{d} / 2}|\mathbf{W}|^{-1 / 2} \exp \left\{-\frac{1}{2}[\mathbf{Y}-\mathbf{I}(\mathbf{Z})]^{T} \mathbf{W}^{-1}[\mathbf{Y}-\mathbf{I}(\mathbf{Z})]\right\}
$$

where $\mathbf{I}$ is the vector containing the radiation intensities calculated with the direct problem solution employing the parameters values $\mathbf{Z}$, at the same positions and polar angles of the measurements $\mathbf{Y}$.

The inverse problem solution can be seen as the values of $\mathbf{Z}$ that maximizes the likelihood function given by Eq. (3), which can be achieved with the minimization of the argument of the exponential function, leading to the following objective function:

$$
S(\mathbf{Z})=[\mathbf{Y}-\mathbf{I}(\mathbf{Z})]^{T} \mathbf{W}^{-1}[\mathbf{Y}-\mathbf{I}(\mathbf{Z})]
$$


Hence, the minimization of the objective function given by Eq. (4) leads to the sought estimates of the radiative properties, hereafter called $\hat{\mathbf{Z}}$.

Due to the unavoidable presence of experimental errors, it is necessary to assess the uncertainty of the parameters estimates. Considering a second-order Taylor expansion of the objective function at the estimate $\hat{\mathbf{Z}}$, and considering the Gauss approximation for the Hessian matrix, one obtains the following expression for the covariance matrix related to the parameters estimates [6]:

$$
\mathbf{V}=\left[\mathbf{J}^{T} \mathbf{W}^{-1} \mathbf{J}\right]^{-1}
$$

where $\mathbf{J}$ is the Jacobian matrix, whose elements are given by:

$$
J_{i j}=\frac{\partial I_{i}}{\partial Z_{j}}, \quad i=1,2, \ldots, N_{d}, \quad j=1,2, \ldots, N_{p}
$$

where $N_{p}$ is the number os parameters, i.e. the dimension of vector $\mathbf{Z}$.

Equation (5) is traditionally employed to define the estimates confidence region, with:

$$
S(\mathbf{Z})-S(\hat{\mathbf{Z}})=[\mathbf{Z}-\hat{\mathbf{Z}}]^{T} \mathbf{V}^{-1}[\mathbf{Z}-\hat{\mathbf{Z}}] \leq \chi_{N_{p}}^{2}
$$

where $\chi_{N_{p}}^{2}$ is the value of the chi-square distribution with $N_{p}$ degrees of freedom for a given probability (confidence level), defining a hyper-ellipsoid in the parameter space.

Even though this approach is often employed for the construction of confidence regions, it should be highlighted that in nonlinear inverse problems, even when the experimental deviations follow the normal distribution, the parameters uncertainties do not necessarily are normally distributed. Hence, the elliptical confidence region might be a poor approximation $[3,4]$.

Alternatively, considering the objective function is a random variable that follows the chi-square distribution with $N_{d}-N_{p}$ degrees of freedom, the following expression can be obtained employing the $\mathrm{F}$ distribution $[3,4]$ :

$$
S(\mathbf{Z}) \leq S(\hat{\mathbf{Z}})\left(1+\frac{N_{p}}{N_{d}-N_{p}} F_{N_{p}, N_{d}-N_{p}}^{\alpha}\right)
$$

which defines a confidence region, known as likelihood confidence regions, for the estimates $\hat{\mathbf{Z}}$ with $\alpha$ confidence level.

Even though the confidence region constructed with Eq. (8) is also an approximation for nonlinear inverse problems, it does not require it to be elliptical, leading to very good approximations of the true confidence regions according to [4]. The main drawback of this method is that it requires a very large number of points, and, therefore, objective function evaluations, specially for high dimension problems. The main idea brought by Schwaab and co-workers [3] is to minimize this difficulty by employing stochastic methods for the optimization procedure, as these algorithms generally perform a high number of objective function evaluations. In this work, the Differential Evolution algorithm [5] is employed for this purpose. 


\section{Results and Discussion}

In order to evaluate the combination of the likelihood method and the Differential Evolution algorithm for the construction of the estimates confidence regions against the traditionally employed elliptical confidence regions, experimental data with different measurement error levels were simulated with:

$$
Y_{i}=I_{i}\left(\mathbf{Z}_{\text {exact }}\right)+\varepsilon_{i}, \quad \varepsilon_{i} \sim N\left(0, \sigma_{e}\right), \quad i=1,2, \ldots, N_{d}
$$

The test case considered in this work is $\mathbf{Z}_{\text {exact }}=\{1.0,0.5,0.5,0.5\}$, where $N_{d}=20$ measurements were considered, half acquired at $\tau=0$ and half at $\tau=\tau_{0}$, and two different sets of experimental data were simulated with two measurement error levels, $\sigma=0.002$ and $\sigma=0.01$, in order to evaluate the accuracy of the elliptical confidence region with respect to the magnitude of the experimental deviations in this problem. Figures 2(a,b) depict the experimental data for both scenarios.

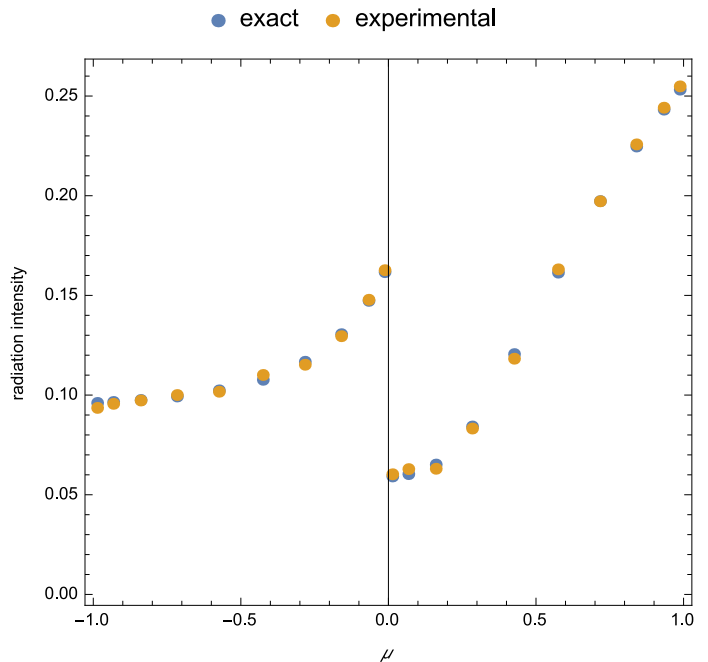

(a)

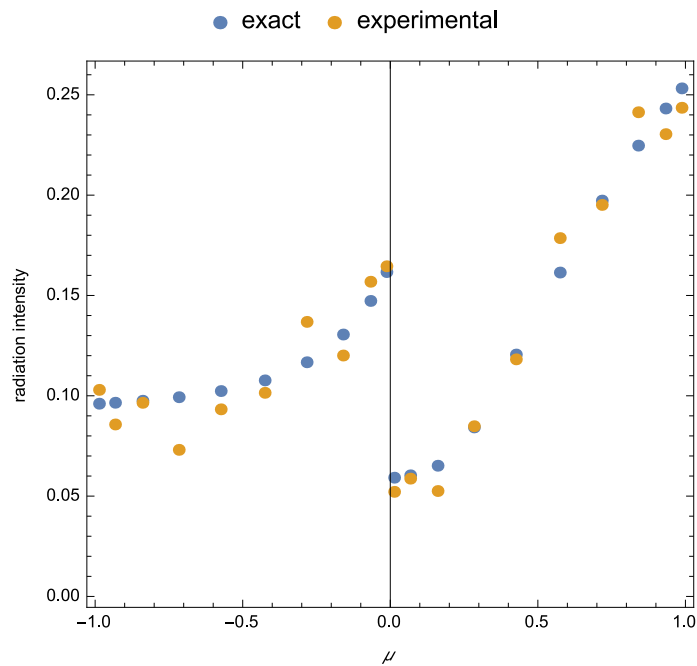

(b)

Figure 2: Simulated experimental data for (a) $\sigma_{e}=0.002$ and (b) $\sigma_{e}=0.01$.

In the Differential Evolution algorithm it was considered the value 0.8 for the weighting factor and 0.9 for the crossover constant, as recommended in [5]. The population size was considered with 320 individuals, much more than the recommendation of ten times the number of parameters, in order to yield a very diversified population and maximize the region where the objective function is evaluated. Finally, 150 generations were considered.

The joint confidence region for this problem is a four-dimensional region. In order to compare the elliptical and the likelihood confidence regions, the results are presented in two dimensions, showing $\tau_{0}$ together with $\omega$ and $\rho_{1}$ together with $\rho_{2}$, which are, in fact, sections of the actual $4 \mathrm{D}$ confidence region.

Figures $3(\mathrm{a}, \mathrm{b})$ show the constructed elliptical confidence region (red line) together with the sampled likelihood confidence region (blue dots), constructed with the objective 
function evaluations performed within the Differential Evolution procedure and Eq. (8), for the case with very small measurement errors $\left(\sigma_{e}=0.002\right)$. These results clearly show that in this case both confidence regions are very similar, indicating that the elliptical region may offer a reliable approximation.

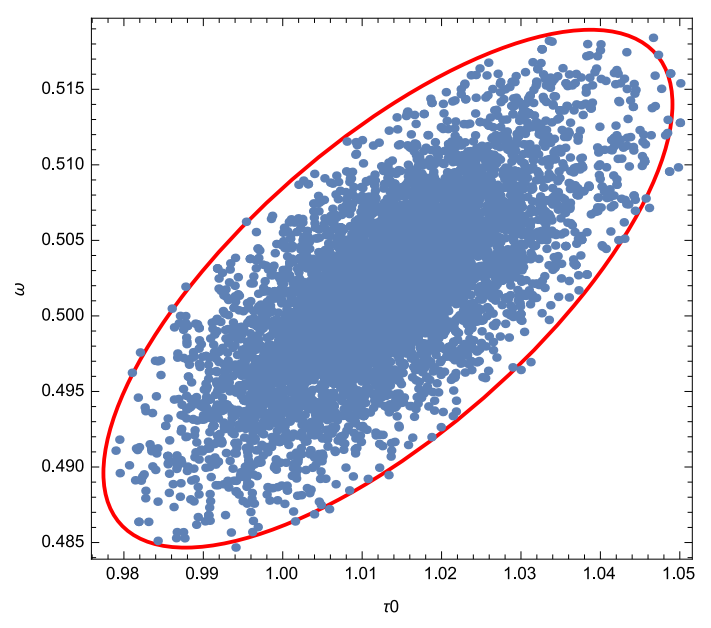

(a)

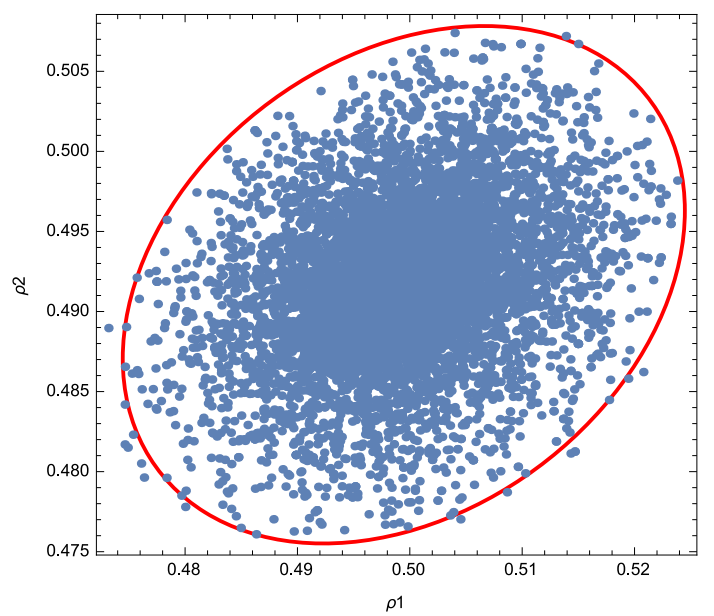

(b)

Figure 3: Estimated elliptical (red line) and likelihood (blue dots) confidence regions for (a) $\tau_{0} \times \omega$ and (b) $\rho_{1} \times \rho_{2}$. Experimental data with $\sigma_{e}=0.002$.

Now, Figures $4(\mathrm{a}, \mathrm{b})$ present similar results for the case with $\sigma_{e}=0.01$. One may first observe that both confidence regions are wider than in the previous case due to higher measurements deviations, as expected. But now the likelihood confidence region is quite different from the elliptical one, which clearly yielded underestimated results, i.e. the elliptical confidence region is unable to identify a relatively large region of parameters values which in fact leads to similar model fits. These results demonstrate that when the experimental errors increase the traditionally employed elliptical confidence region fails to yield reliable results for the inverse radiative transfer problem. Hence, the likelihood method should be preferred, or if the elliptical confidence region is adopted, a previous study should be carried out in order to assess which levels of measurement errors yield acceptable results.

\section{Conclusions}

This work provided the estimation of radiative properties in one-dimensional participating media, employing a combination of the Differential Evolution algorithm with the likelihood method for the construction of confidence regions, making use of the several objective function evaluations performed during the optimization procedure to construct more reliable confidence regions in comparison with the traditionally employed elliptical confidence region. 


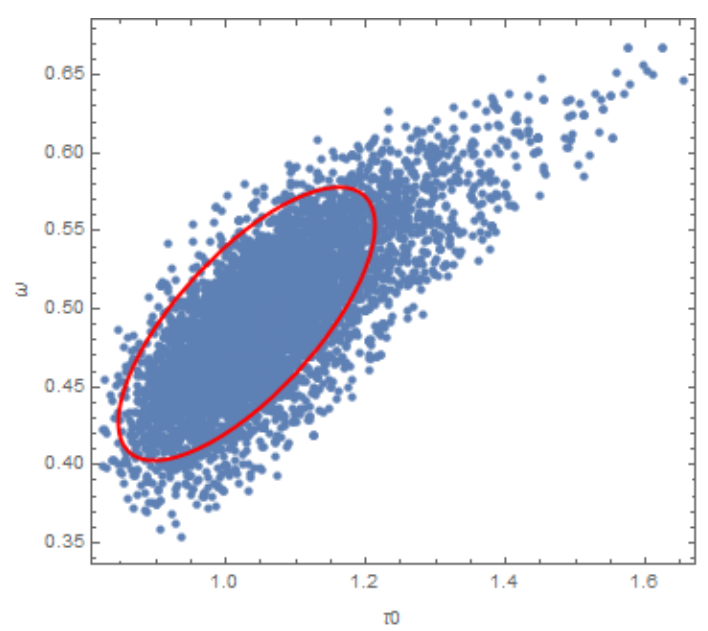

(a)

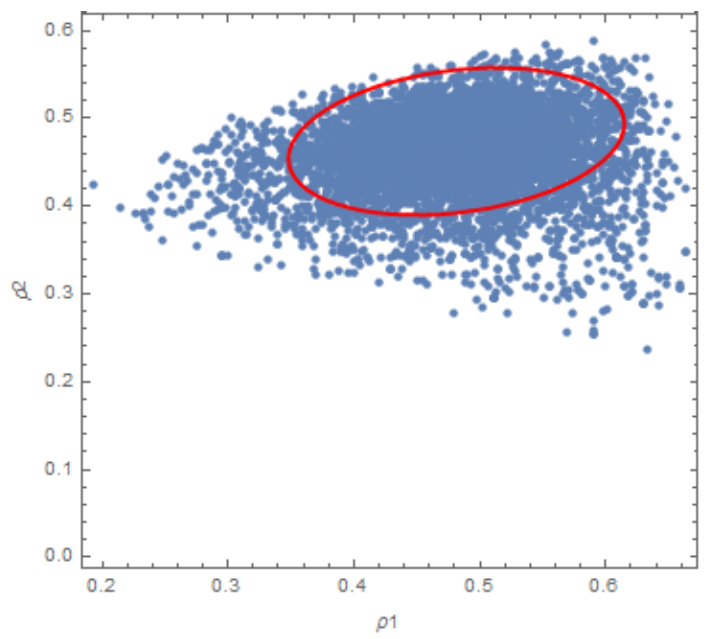

(b)

Figure 4: Estimated elliptical (red line) and likelihood (blue dots) confidence regions for (a) $\tau_{0} \times \omega$ and (b) $\rho_{1} \times \rho_{2}$. Experimental data with $\sigma_{e}=0.01$.

\section{Acknowledgements}

The authors acknowledge the financial support provided by FAPERJ and CNPq.

\section{References}

[1] M. N. Özışı. Radiative transfer and interactions with conduction and convection. Wiley-Interscience, 1973.

[2] A. J. Silva Neto and J. C. Becceneri. Técnicas de inteligência computacional inspiradas na natureza: Aplicação em problemas inversos em transferência radiativa. Notas em Matemática Aplicada, 41, 2009.

[3] M. Schwaab, E. C. Biscaia Jr., J. L. Monteiro, and J. C. Pinto. Nonlinear parameter estimation through particle swarm optimization. Chemical Engineering Science, 63:1542-1552, 2008.

[4] J. R. Donaldson and R. B. Schnabel. Computational experience with confidence regions and confidence intervals for nonlinear least squares. Technometrics, 29(1):67-82, 1987.

[5] R. Storn. On the usage of differential evolution for function optimization. In Conference of the North American Fuzzy Information Processing Society (NAFIPS), IEEE, pages 519-523, Berkeley, 1996.

[6] M. Schwaab and J. C. Pinto. Análise de dados experimentais I. Fundamentos de estatística e estimação de parâmetros. E-papers, Rio de Janeiro, 2007. 